\title{
Postnatal human enteric neuronal progenitors can migrate, differentiate, and proliferate in embryonic and postnatal aganglionic gut environments
}

\author{
Lily S. Cheng ${ }^{1,2}$, Ryo Hotta', Hannah K. Graham', Jaime Belkind-Gerson³, Nandor Nagy ${ }^{1,4}$, and Allan M. Goldstein ${ }^{1}$
}

BACKGROUND: Enteric neural stem/progenitor cells (ENSCs) offer an innovative approach to treating Hirschsprung disease (HSCR) and other enteric neuropathies. However, postnatalderived human ENSCs have not been thoroughly characterized and their behavior in the embryonic and postnatal intestinal environment is unknown.

METHODS: ENSCS were isolated from the intestines of 25 patients undergoing bowel resection, including 7 children with HSCR. Neuronal differentiation and proliferation of ENSCs from submucosal and myenteric plexuses from patients with and without HSCR were characterized. ENSC migration and differentiation were studied following transplantation into embryonic chick neural crest, embryonic chick hindgut, and postnatal mouse aganglionic colon.

RESULTS: The proliferative and neurogenic potential of ENSCs from HSCR intestine is equivalent to that of non-HSCR controls. Similarly, no difference was observed between myenteric- and submucosal-derived ENSCs. Postnatal ENSCs transplanted to embryonic neural crest pathways and to aneural hindgut migrate normally and differentiate into appropriate neural crest-derived cell types. ENSCs in postnatal mouse aganglionic colon differentiate into neurons and glia both ex vivo and in vivo.

CONCLUSIONS: ENSCs isolated from the postnatal intestine of patients with and without HSCR can behave like embryonic neural crest-derived cells. These results support the feasibility of cell-based therapy for future treatment of neurointestinal disease.

$\mathrm{T}$ he enteric nervous system (ENS), the largest division of the autonomic nervous system is a vast network of neurons and glia within the wall of the gastrointestinal (GI) tract. It plays an essential role in regulating normal GI functions including motility, secretion, absorption, and immune function (1). Abnormalities of the ENS cause a broad spectrum of functional GI disorders including gastroparesis, esophageal achalasia, anal achalasia, intestinal pseudo-obstruction, and slow-transit constipation. Hirschsprung disease (HSCR) is the archetypal disease of the ENS and is caused by the failure of neural crest-derived cells to complete migration in the GI tract, leaving variable lengths of distal intestine aganglionic (2). Despite surgery, which consists of resecting the aganglionic segment, patients often continue to suffer from persistent problems with chronic constipation, fecal incontinence, and life-threatening enterocolitis $(3,4)$. Neural stem/progenitor cell therapy offers the potential to rebuild the ENS in HSCR and other enteric neuropathies (5-7).

Several groups have reported successful isolation and propagation of human postnatal gut-derived enteric neural stem/progenitor cells (ENSCs) in culture as floating neurospheres (8-10). These cells can be expanded exponentially and are capable of differentiating into neurons and glia (11). Human ENSCs have been isolated from adults up to 84 y old (10), from small and large intestine, including the aganglionic segment of patients with HSCR (12), from full thickness bowel, and from mucosal biopsies (13). However, the proliferative and neurogenic potential of ENSCs derived from these various sources have not been fully characterized. Studies in mice harboring mutations in Ednrb or Ret, common culprits in the pathogenesis of HSCR, suggest that there is no alteration in the proliferation or differentiation of ENSCs derived from normal and diseased animals (1416). However, the properties of ENSCs derived from human patients with HSCR have not been compared with ENSCs derived from patients without neurointestinal disease, and this is essential in establishing the feasibility of autologous cell therapy in the future. In addition, it has been reported that murine neurospheres derived from the myenteric plexus (MP) contain more neural crest-derived cells and produce more neurons than neurospheres derived from the submucosal plexus (SP) (17), but cells isolated from human SP have conversely shown a greater propensity to form neurospheres (18). The composition and neurogenic potential of human MP-and SP-derived neurospheres have not been compared.

\footnotetext{
The last two authors are co-corresponding authors.

'Department of Pediatric Surgery, Massachusetts General Hospital, Harvard Medical School, Boston, Massachusetts; ${ }^{2}$ Department of Surgery, University of California San Francisco, San Francisco, California; ${ }^{3}$ Department of Pediatrics, Massachusetts General Hospital, Harvard Medical School, Boston, Massachusetts; ${ }^{4}$ Department of Anatomy, Histology and Embryology, Faculty of Medicine, Semmelweis University, Budapest, Hungary. Correspondence: Allan M. Goldstein (agoldstein@partners.org) Received 9 June 2016; accepted 30 November 2016; advance online publication 1 March 2017. doi:10.1038/pr.2017.4
} 
Prior studies have shown that human postnatal-derived ENSCs transplanted into aganglionic embryonic mouse hindgut can differentiate into neurons and begin to regulate gut function $(12,19)$. It has also been reported that human postnatal-derived ENSCs transplanted to neurologically-damaged postnatal mouse colon can improve contractility despite limited engraftment (20), but most studies have focused on transplantation of postnatal precursors to embryonic gut. It is not known what other effects the embryonic environment might have on postnatal-derived ENSCs or how ENSC behavior in the embryonic gut may differ from that in the postnatal environment.

The goals of this study were to characterize human postnatal-derived ENSCs from patients with and without HSCR, to compare neurospheres derived from the myenteric and submucosal plexuses, and to study these cells in embryonic and postnatal environments. Our results yield valuable new information about human postnatal-derived ENSCs and their potential to treat neurointestinal diseases.

\section{METHODS}

Animals

White Leghorn chicken (Gallus gallus) eggs were obtained from commercial breeders and maintained at $37^{\circ} \mathrm{C}$ in a humidified incubator. Mice used in this study were either 3-wk-old C57BL6 mice (Jackson Lab, Bar Harbor, ME) or 3-wk-old homozygous Ednrb ${ }^{\text {tm IYwa }}\left(E_{\text {Ednrb }}{ }^{-/-}\right)$ mice on a hybrid C57BL6/J-129Sv background (B6;129-Ednrb ${ }^{\text {tm1Ywa/J; }}$ Jackson Labs). Ednrb ${ }^{-/-}$mice, characterized by distal colonic aganglionosis and a white coat color, are a model for human HSCR (21). Animal protocols were approved by the Massachusetts General Hospital Institutional Animal Care and Use Committee.

\section{Human Tissue Samples}

With approval from the Massachusetts General Hospital Institutional Review Board, intestinal tissue was obtained from 25 patients undergoing bowel resections, including normally ganglionated proximal colon from 7 patients with HSCR. Ages ranged from 0-24 y and tissue was obtained from small intestine, colon, or appendix.

\section{Isolation and Propagation of ENSC}

To isolate ENSCs, the muscularis propria-containing the longitudinal muscle, circular muscle, and MP-was separated from the underlying submucosa using fine forceps (Figure 1a). The mucosa was separated from the layer containing the submucosa, muscularis mucosa, and SP by gentle scraping with a surgical scalpel blade (Figure 1b). The mucosa was discarded and the submucosal layer containing the SP and the muscularis layer containing the MP were processed separately.

Murine ENSCs were isolated from the colon of 3-wk-old C57BL6 mice and propagated in culture as neurospheres (14). Human ENSCs were isolated and propagated in culture as neurospheres based on previously published protocols $(9,10)$. Tissue was finely minced into $1 \mathrm{~mm}$ pieces and enzymatically dissociated with dispase $(250 \mu \mathrm{g} / \mathrm{ml}$; StemCell Technologies, Vancouver, BC) and collagenase XI (1 mg/ml; Sigma Aldrich, St. Louis, MO) at $37^{\circ} \mathrm{C}$ for $90 \mathrm{~min}$. Single cells were isolated by filtration through a $70 \mu \mathrm{m}$ filter and plated at $50,000 \mathrm{cells} / \mathrm{ml}$ in a $10-\mathrm{cm}^{2}$ flask in cell media with human proliferation media, consisting of Neurocult Human Basal Medium (StemCell Technologies) supplemented with 10\% Neurocult Human Proliferation Supplement (StemCell Technologies), $20 \mathrm{ng} / \mathrm{ml}$ epidermal growth factor (StemCell Technologies), $20 \mathrm{ng} / \mathrm{ml}$ basic fibroblast growth factor (StemCell Technologies), $0.0002 \%$ Heparin (StemCell Technologies), $50 \mu \mathrm{g} /$ $\mathrm{ml}$ metronidazole (Sigma Aldrich), $2 \mu \mathrm{l} / \mathrm{ml}$ Primocin (Invivogen, Carlsbad, CA), in a $1: 1$ mixture with mouse conditioned media (supernatant from cultured murine ENSCs). After $7 \mathrm{~d}$, primary cell aggregates were dissociated with Accutase (StemCell Technologies) at $37^{\circ} \mathrm{C}$ for $30 \mathrm{~min}$ and replated at 50,000 cells $/ \mathrm{ml}$ in a $96-$ well round bottom plate (Corning, Kennebunk, ME), which was centrifuged at $480 \mathrm{~g}$ for $2 \mathrm{~min}$ to encourage cell aggregation. Secondary neurospheres formed after $7 \mathrm{~d}$ in culture (Figure 1c-e). Half the media was replaced every $7 \mathrm{~d}$. Neurospheres were kept in culture for up to $30 \mathrm{~d}$.

\section{Immunohistochemistry}

Tissue preparation and immunohistochemistry were performed as described (22). Cells and tissues were fixed in $4 \%$ paraformaldehyde. For cryosection, tissue was incubated in $15 \%$ sucrose at $4^{\circ} \mathrm{C}$ overnight, and then in $15 \%$ sucrose containing $7.5 \%$ gelatin at $37^{\circ} \mathrm{C}$ for $1 \mathrm{~h}$ and rapidly frozen at $-50^{\circ} \mathrm{C}$. Frozen sections were cut at 12 $\mu \mathrm{m}$ thickness with a Leica CM3050 S cryostat (Leica, Buffalo Grove, IL). For immunohistochemistry, cells and tissues were permeabilized with $0.1 \%$ Triton X-100 and blocked with $10 \%$ donkey serum for $30 \mathrm{~min}$. Primary antibodies included mouse antihuman neuronal protein (HU; 1:100; Molecular Probes, Eugene, OR), mouse antimelanocyte antibody (MITF; 1:100; generous gift from Malkiel Cohen and Rudolf Jaenisch), mouse antineuronal class III $\beta$-tubulin (Tuj1; 1:500; Covance, Dedham, MA), mouse anti-E-cadherin (ECad; 1:200; BD Biosciences, San Jose, CA), rabbit anti-p75 neurotrophin receptor (p75; 1:500; Promega, Madison, WI), rabbit anti-S100 calciumbinding protein B (S100; 1:100; NeoMarkers, Fremont, CA), rabbit anticalretinin (CR; 1:200; Invitrogen), and rabbit antismooth muscle actin (SMA; 1:200; Abcam, Cambridge, MA). Secondary antibodies included donkey antirabbit Alexa Fluor 546 and donkey antimouse Alexa Fluor 488 and 546 (Life Technologies). Alexa-Fluor 488-conjugated antihuman nuclei antibody (HUNUC; 1:50; Millipore, Temecula, CA) was used to identify human-derived cells. Cell nuclei were stained with 4,6-diamidino-2-2-phenylindole (DAPI) (Vector Labs, Burlingame, CA). Images were taken using a Nikon Eclipse 80i microscope or a Nikon A1R laser scanning confocal microscope (Nikon, Melville, NY).

\section{Characterization of Neurospheres}

Secondary neurospheres were dissociated with Accutase incubation for $30 \mathrm{~min}$ at $37^{\circ} \mathrm{C}$. To quantify the number neural crest-derived cells in the neurosphere, cells were centrifuged $(800 \mathrm{~g}$ for $2 \mathrm{~min}$; Shandon Cytospin 3) onto poly-L-lysine slides and neural crest-derived cells visualized with $\mathrm{p} 75$ immunoreactivity. To quantify the neurogenic and proliferative potential of ENSC, dissociated secondary neurospheres were replated at 5,000 cells $/ \mathrm{ml}$ in either human or mouse differentiation media, consisting of Neurocult Human or Mouse Basal Medium (StemCell Technologies) supplemented with 10\% Neurocult Human or Mouse Differentiation Supplement (StemCell Technologies), 10\% fetal bovine serum, and $100 \mathrm{U} / \mathrm{ml}$ penicillin-streptomycin (Life Technologies), on glass-bottom chamber slides coated with $20 \mu \mathrm{g} /$ $\mathrm{ml}$ fibronectin (Biomedical Technologies, Ward Hill, MA). Cells were cultured for $7 \mathrm{~d}$ and $10 \mu \mathrm{mol} / \mathrm{l}$ EdU was added to the culture media $24 \mathrm{~h}$ prior to fixation. EdU incorporation was detected using the Click-iT EdU Imaging Kit (Invitrogen, Carlsbad, CA).

To compare cells from patients with and without HSCR, MP-derived ENSCs from HSCR patients ( $n=3$; age range: from $11 \mathrm{~d}$ to $9 \mathrm{y}$ old) were compared with colonic MP-derived ENSCs from patients of similar ages ( $n=3$; age range: from 1 mo to 6 y old). To compare MPto SP-derived cells, cell density, and proliferation were normalized to MP for each patient $(n=9)$. ENSC characterization was repeated at least two times for each human sample and 10 nonoverlapping, representative images were taken from each preparation.

\section{Transplantation to Embryonic Chick Neural Crest}

Using fine forceps, somites 3-5 were extirpated from the right side of an E2 (18 somite-stage) chick embryo and a human neurosphere was introduced into this space adjacent to the vagal neural tube (Figure 2a). Recipients were sacrificed at $12 \mathrm{~h}(n=3), 24 \mathrm{~h}(n=3)$, $48 \mathrm{~h}(n=3)$, and $96 \mathrm{~h}(n=3)$ post-transplantation.

\section{Transplantation to Aganglionic Embryonic Chick Hindgut}

As previously described (14), neurospheres were transplanted to the isolated hindgut of E5 or E6 chick embryos (Figure 3a). Hindgut tissue was cultured on the chorioallantoic membrane of an embryonic day 9 (E9) host chick for $7 \mathrm{~d}(n=9$; Figure $3 \mathbf{b})$, then processed for immunofluorescence. 


\section{Articles | Chenget al.}
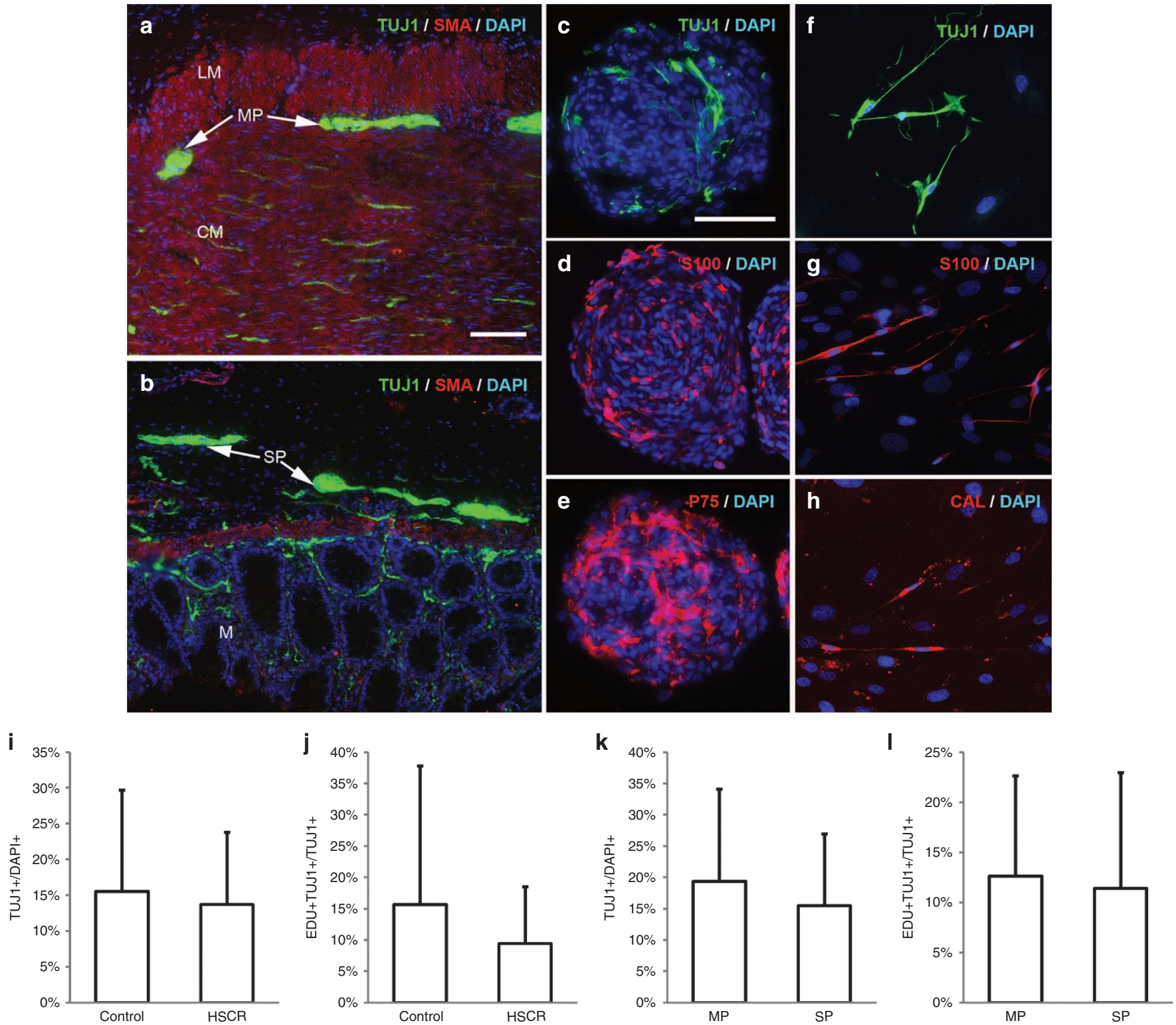

Figure 1. Neurospheres can be generated from myenteric (MP) and submucosal (SP) plexus of postnatal human intestine. Full thickness intestine was obtained from patients undergoing bowel resection. Tissue was separated into a layer containing longitudinal muscle, circular muscle, and MP (a) and a layer containing submucosa, muscularis mucosa, and SP (b). Cells from both layers form floating neurospheres containing neurons (TUJ1, green; c), glia (S100, red; $\mathbf{d}$ ), and neural crest-derived cells (P75, red; e). ENSCs from dissociated neurospheres give rise to neurons (TUJ1, green; f), glia (S100, red; $\mathbf{g}$ ), and neuronal subtypes, including calretinin-immunoreactive neurons (CAL, red; $\mathbf{h}$ ). ENSCs from patients with HSCR do not differ significantly in neuronal density (i) or proliferation (j) compared with patients without HSCR. ENSCs from MP and SP have equivalent neuronal density (k) and proliferation (I). Cell nuclei are counterstained with DAPI (blue; $\mathbf{a}-\mathbf{h}$ ). Scale bar in $\mathbf{a}=100 \mu \mathrm{m}$ and applies to $\mathbf{a}$, b. Scale bar in $\mathbf{c}=100 \mu \mathrm{m}$ and applies $\mathbf{c}-\mathbf{h}$. CAL, calretinin; CM, circular muscle; DAPI, 4,6-diamidino-2-2-phenylindole; ENSCs, Enteric neural stem/progenitor cells; HSCR, Hirschsprung disease; LM, longitudinal muscle; $\mathrm{M}$, mucosa; MP, myenteric plexus; SMA, smooth muscle actin; SP, submucosal plexus.

Transplantation to Aganglionic Postnatal Mouse Hindgut Ex Vivo As previously described (14), neurospheres were cocultured with the isolated muscularis propria of aganglionic colon from $E d n r b^{-/-}$mice $(n=6)$ or ganglionic colon from C57BL6 mice $(n=4)$. Each neurosphere was placed within a small pocket in the muscularis propria and the tissue was suspended as a caternary culture using a piece of filter paper with a rectangular window. The preparation was cultured for 7 $\mathrm{d}$ in human differentiation media with $10 \mu \mathrm{mol} / \mathrm{l}$ EdU added to the culture media $24 \mathrm{~h}$ prior to fixation.

Transplantation to Aganglionic Postnatal Mouse Hindgut In Vivo Neurospheres were transplanted to the aganglionic distal colon of 3 -wk-old $\mathrm{Ednrb}^{-/-}$mice $(n=3)(14)$. A perineal incision was made to expose the colon just above the anus and a neurosphere was placed within a small pocket in the muscularis propria. The pocket was closed with 8-0 nonabsorbable monofilament suture and the skin was closed with 5-0 nonabsorbable braided suture. Recipients received $5 \mathrm{mg} / \mathrm{kg}$ of tacrolimus (Sigma Aldrich) daily via subcutaneous injection. Two mice survived to sacrifice $5 \mathrm{~d}$ following transplantation.

\section{Statistics}

Numbers of cells were counted using ImageJ software (National Institutes of Health, Bethesda, Maryland). Data are presented as mean \pm SD. Results were compared using Student's $t$-test for parametric data and Mann-Whitney $U$-test for nonparametric data. Statistical significance was considered at $P<0.05$. Statistical analysis was performed using JMP, Version 12 (SAS Institute, Cary, NC). 

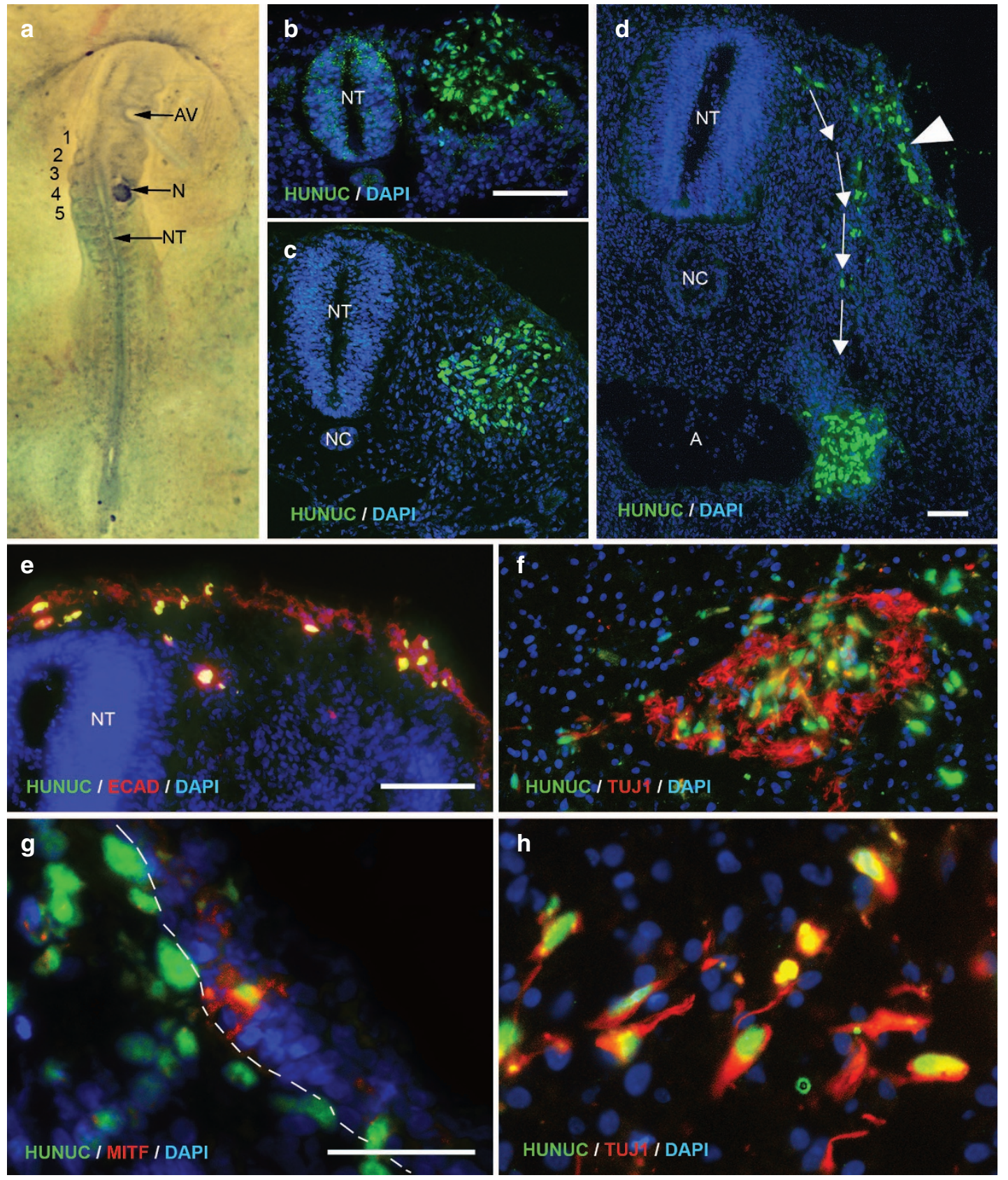

Figure 2. Human ENSCs transplanted into embryonic chick migrate and differentiate along normal neural crest cell pathways. Human neurospheres were transplanted adjacent to the E2 chick neural tube at somite level 3-5 (a). Transplanted cells express a human nuclear marker (HUNUC, green) and are seen after $12 \mathrm{~h}$ (b) and $24 \mathrm{~h}$ (c). At $48 \mathrm{~h}$, transplanted cells are observed migrating dorsolaterally (arrowhead; d) and ventromedially (arrows; d). The dorsolateral cells incorporate into the chick ectoderm (ECAD, red; $\mathbf{e}$ ) and express MITF (red), a human melanocyte marker (g; dashed line marks epidermal-mesenchymal border). Ventromedial cells migrate toward the foregut and differentiate into neurons (TUJ1, green; $\mathbf{f}, \mathbf{h}$ ). Cell nuclei are counterstained with DAPI (blue; $\mathbf{b}-\mathbf{h}$ ). Scale bar in $\mathbf{b}=100 \mu \mathrm{m}$ and applies to $\mathbf{b}$, c. Scale bar in $\mathbf{d}=100 \mu \mathrm{m}$. Scale bar in $\mathbf{e}=50 \mu \mathrm{m}$ and applies to e, f. Scale bar in $\mathbf{g}=50$ $\mu \mathrm{m}$ and applies to $\mathbf{g}$, $\mathbf{h}$. A, aorta; AV, auditory vesicle; DAPI, 4,6-diamidino-2-2-phenylindole; ENSCs, Enteric neural stem/progenitor cells; NC, notochord; N, neurosphere; NT, neural tube.

\section{RESULTS}

\section{Postnatal Human Enteric Neurospheres from HSCR and Control} Intestine do not Differ in their Proliferative and Neurogenic Potential

Tissue was collected from the small intestine, colon, or appendix of 25 patients (Table 1). Neurospheres were successfully generated from 21 patients; 3 samples generated no neurospheres and 1 sample was discarded due to bacterial contamination. Following initial dissociation, primary cell yield was $7.3 \pm 4.8$ million cells per $\mathrm{cm}^{2}$ of tissue. After $7 \mathrm{~d}$ in selective culture, this amount of starting material yields an average of $25 \pm 23$ neurospheres. Differentiation of dissociated neurospheres yielded an average of $17.4 \pm 9.9 \%$ neurons, as determined by Tuj1 immunoreactivity. Neuronal proliferation, determined by 


\section{Articles $\mid$ Chenget al.}
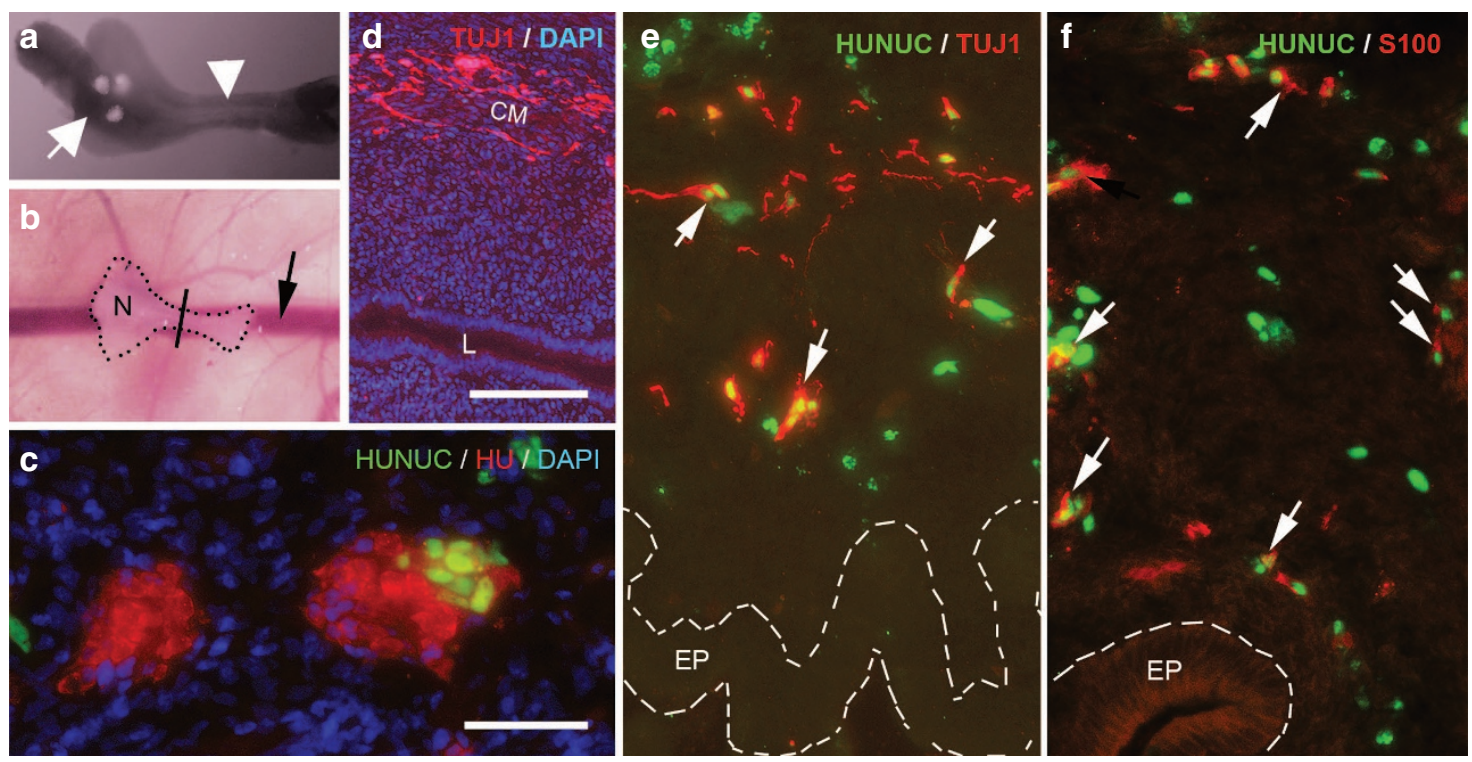

Figure 3. Human ENSCs migrate and differentiate into neurons and glia in the embryonic chick hindgut. Human ENSC were transplanted to E5 (preganglionic) or E6 (ganglionic) chick hindgut. Neurospheres were transplanted into the cecum (a; arrow marks neurospheres) and the hindgut (a; arrowhead) was cultured on the chorioallantoic membrane (CAM) of an E9 host chick for $7 \mathrm{~d}$ (b; $\mathrm{N}$ highlights site of neurosphere transplantation in the cecum, arrow marks chorioallantoic membrane vessel, line denotes the approximate gut level shown in $\mathbf{c}-\mathbf{f}$ ). When neurospheres are transplanted to ganglionic E6 hindgut, enteric ganglia contain both HU+ (red) / HUNUC- and HU+ (red) / HUNUC+ (green) cells, consistent with chick-and human-derived chimeric ganglia, respectively (c). When neurospheres are transplanted to preganglionic E5 hindgut, Tuj1+ neurons migrate extensively along the wall of the gut (d). Costaining with HUNUC confirms that neurons (TUJ1, red; e) and glia (S100, red; $\mathbf{f}$ ) are derived from transplanted human cells. Cell nuclei are counterstained with DAPI (blue; c-d). Scale bar in c $=50 \mu \mathrm{m}$. Scale bar in $\mathbf{d}=100 \mu \mathrm{m}$ and applies d-f. CM, circular muscle; DAPI, 4,6-diamidino-2-2-phenylindole; ENSCs, enteric neural stem/progenitor cells; EP, epithelium; L, lumen; $N$, neurosphere.

the proportion of Tuj1 immunoreactive cells with EdU uptake, averaged $12.0 \pm 8.9 \%$.

Neuronal differentiation and proliferation did not differ significantly in ENSCs derived from the normally ganglionated colon obtained from the proximal resection margin of patients with HSCR ( $13.7 \pm 10.1 \%$ neurons with $9.4 \pm 9.1 \%$ proliferating) compared with those derived from colon of age-matched patients without HSCR $(15.5 \pm 14.2 \%$ neurons with $15.6 \pm 22.2 \%$ proliferating; Figure 1i,j). Neuronal differentiation and proliferation also did not differ significantly with ENSCs derived from large $(16.7 \pm 3.5 \%$ neurons with $11.9 \pm 11.7 \%$ proliferating) and small intestine $(23.1 \pm 18.2 \%$ neurons with $5.8 \pm 3.2 \%$ proliferating; data not shown), nor did it correlate with the age of the patient $\left(R^{2}=0.23\right.$ for neuronal differentiation; $\mathrm{R}^{2}=0.11$ for neuronal proliferation).

\section{MP- and SP-derived ENSCs have Similar Proliferative and Neurogenic Potential}

In human postnatal-derived ENSCs, neuronal differentiation and proliferation of SP-derived ENSCs $(15.5 \pm 11.4 \%$ neurons with $11.4 \pm 11.5 \%$ proliferating) did not differ significantly when compared with MP-derived ENSCs $(19.3 \pm 14.8 \%$ neurons with $12.6 \pm 10.0 \%$ proliferating; Figure $1 \mathrm{k}-1$ ). However, average neurosphere diameter was significantly greater among MP neurospheres $(189 \pm 11$ vs. $159 \pm 7 \mu \mathrm{m}, P<0.05)$. More neurospheres were generated from SP tissue, although this did not reach statistical significance ( $16 \pm 14$ vs. $9 \pm 10$ neurospheres per $\mathrm{cm}^{2}$ of tissue) due to the large variability.

We also examined the properties of postnatal-derived ENSCs isolated from both plexuses of the mouse intestine.
As with human ENSCs, average neurosphere diameter was greater for mouse MP neurospheres (200 \pm 28 vs. $157 \pm 16 \mu \mathrm{m}$, $P<0.05)$ and there was no significant difference in the number of neurospheres generated from MP and SP $(71 \pm 40$ vs. $42 \pm 23$ neurospheres per mouse colon). The number of neural-crest derived cells, identified by immunoreactivity for the neural crest cell marker p75, was greater in MP neurospheres $(35.5 \pm 16.9 \%)$ than SP neurospheres $(12.2 \pm 9.8 \%, P<0.05$; Supplementary Figure S1c online). However, neuronal differentiation and proliferation of MP ENSCs $(48.8 \pm 18.2 \%$ neurons with $7.8 \pm 7.0 \%$ proliferating) did not differ significantly when compared with SP ENSCs $(48.3 \pm 18.2 \%$ neurons with $4.5 \pm 3.2 \%$ proliferating; Supplementary Figure S1d,e online).

\section{Human Postnatal ENSCs Migrate along Normal Neural Crest Pathways in the Chick Embryo}

To examine the behavior of human postnatal-derived ENSCs in the early embryonic environment, neurospheres were transplanted adjacent to the vagal region of the neural crest of embryonic day 2 (E2) chicks (Figure 2a). This location was chosen for the transplant as it corresponds to the axial level of the neural crest that gives rise to the neurons and glia that will form the ENS (2). Cells expressing a human nuclear marker were identified 12-96h following transplantation (Figure 2b,c). After $48 \mathrm{~h}$, transplanted cells migrated along the two expected paths: ventromedial and dorsolateral (Figure 2d). Human ENSCs migrated ventromedially toward the foregut and expressed the neuronal marker, Tuj1, indicating neuronal differentiation (Figure 2f, h). Human ENSCs that 
migrated dorsolaterally incorporated into chick ectoderm, which is immunoreactive for the chick-specific ectoderm marker, E-cadherin (Figure 2e). These ENSCs included cells positive for the human-specific melanocyte marker, MITF, confirming melanocytic differentiation (Figure 2g).

Human ENSCs Transplanted to Embryonic Chick Hindgut Exhibit Intramural Migration and Neuroglial Differentiation

To study the behavior of human postnatal-derived ENSCs in the embryonic gut, neurospheres were transplanted to the hindgut of embryonic day 5 (E5) or 6 (E6) chicks and cultured on host chick chorioallantoic membrane (Figure 3a,b). Since endogenous neural crest cells reach the chick hindgut at E6, the E5 hindgut is physiologically aneural. ENSC transplantation into E6 hindgut, which contains endogenous crest-derived cells, leads to formation of chimeric enteric ganglia, containing both human-derived enteric neurons ( $\mathrm{HU}+$ ) and endogenous chick neurons (Figure 3c). Seven d after transplantation to E5 hindgut, Tuj1+ cells were present circumferentially within the gut wall (Figure 3d). Transplanted cells expressing a human nuclear marker costained with Tuj1, indicating neuronal differentiation (Figure 3e), and S100, a marker of glial differentiation (Figure 3f).

\section{Human ENSCs Transplanted to Postnatal Mouse Colon Differentiate into Neurons and Glia Ex Vivo and In Vivo}

To study the behavior of human postnatal-derived ENSCs in the postnatal gut, neurospheres were transplanted ex vivo onto the muscularis propria of explanted colon from HSCR model $\left(E d r n b^{--}\right)$or normal (C57BL6) mice. After $7 \mathrm{~d}$, transplanted ENSCsmigratedalongthemuscleinbothaganglionic(Figure4a) and ganglionic colon with a similar distance of migration $(246 \pm 134$ vs. $192 \pm 74 \mu \mathrm{m})$. In both aganglionic and ganglionic colon, transplanted cells expressed markers of neuronal (Tuj1, HU; Figure 4a-b) and glial (S100; Figure 4c) differentiation. Transplanted Tuj1+ neurons coexpressed synaptophysin (SYP; Figure 4d), suggesting synapse formation, and incorporated EdU (Figure 4e), indicative of cell proliferation after transplantation.

Human ENSCs were next transplanted into the aganglionic distal colon of HSCR mice in vivo. After $5 \mathrm{~d}$, human ENSCs were present in the gut wall, close to the site of transplantation (Figure 4f). Transplanted ENSCs gave rise to neurons (Tuj1; Figure 4g) and glia (S100; Figure 4h) within the aganglionic gut in vivo.

\section{DISCUSSION}

Postnatal gut-derived ENSCs represent a promising source for cell-based therapy in neurointestinal diseases, including HSCR. Characterizing the developmental potential and neurogenicity of human enteric neuronal progenitors is essential in order to develop cell therapy as a viable approach for treating enteric neuropathies. In this study, we observed a high degree of variability in the proliferation and differentiation of ENSCs from different patients. This likely results from a number of patient-specific factors, including the underlying
Table 1. Sources for enteric neural stem cells in order by patient age

\begin{tabular}{|c|c|c|c|c|}
\hline Age & Sex & Diagnosis & Operation & Tissue \\
\hline $2 d$ & M & Jejunal atresia & Small bowel resection & Jejunum \\
\hline $11 d$ & $\mathrm{~F}$ & $\begin{array}{l}\text { Hirschsprung } \\
\text { disease }\end{array}$ & Pullthrough & Colon \\
\hline $1 \mathrm{mo}$ & M & Colonic stricture & Colon resection & Colon \\
\hline $1 \mathrm{mo}$ & M & $\begin{array}{l}\text { Hirschsprung } \\
\text { disease }\end{array}$ & Pull through & Colon \\
\hline $2 \mathrm{mo}$ & M & $\begin{array}{l}\text { Hirschsprung } \\
\text { disease }\end{array}$ & Pull through & Colon \\
\hline $2 \mathrm{mo}$ & M & $\begin{array}{l}\text { Hirschsprung } \\
\text { disease }\end{array}$ & Pull through & Colon \\
\hline $9 \mathrm{mo}$ & M & $\begin{array}{c}\text { Anorectal } \\
\text { malformation }\end{array}$ & Colostomy closure & Colon \\
\hline $2 y$ & M & $\begin{array}{l}\text { Hirschsprung } \\
\text { disease }\end{array}$ & Pullthrough (redo) & Colon \\
\hline $4 y$ & M & Appendicolith & Appendectomy & Appendix \\
\hline $6 y$ & $\mathrm{~F}$ & $\begin{array}{c}\text { Anorectal } \\
\text { malformation }\end{array}$ & Colostomy closure & Colon \\
\hline $9 y$ & M & $\begin{array}{l}\text { Hirschsprung } \\
\text { disease }\end{array}$ & Pullthrough & Colon \\
\hline $11 y$ & $\mathrm{~F}$ & $\begin{array}{l}\text { Chronic } \\
\text { abdominal pain }\end{array}$ & Appendectomy & Appendix \\
\hline $12 y$ & $\mathrm{~F}$ & $\begin{array}{l}\text { Chronic } \\
\text { appendicitis }\end{array}$ & Appendectomy & Appendix \\
\hline $13 y$ & M & $\begin{array}{l}\text { Chronic } \\
\text { abdominal pain }\end{array}$ & Appendectomy & Appendix \\
\hline $14 y$ & M & $\begin{array}{l}\text { Hirschsprung } \\
\text { disease }\end{array}$ & Pullthrough (redo) & Colon \\
\hline $14 y$ & M & Ulcerative colitis & Colectomy & Colon \\
\hline $14 y$ & M & Ulcerative colitis & $\begin{array}{c}\text { Restorative } \\
\text { proctocolectomy (redo) }\end{array}$ & Ileum \\
\hline $15 y$ & M & Crohn's disease & Ileostomy closure & Ileum \\
\hline $16 y$ & M & Ulcerative colitis & $\begin{array}{l}\text { Restorative } \\
\text { proctocolectomy }\end{array}$ & Ileum \\
\hline $16 y$ & M & $\begin{array}{l}\text { Ulcerative colitis, } \\
\text { nonrotation }\end{array}$ & Appendectomy & Appendix \\
\hline $16 y$ & M & Crohn's disease & Ileostomy revision & Ileum \\
\hline $17 y$ & M & Crohn's disease & Ileocecal resection & Ileum \\
\hline $17 y$ & M & Crohn's disease & Ileocecal resection & Colon \\
\hline $21 y$ & M & Ulcerative colitis & Ileostomy closure & Ileum \\
\hline $24 y$ & $\mathrm{~F}$ & $\begin{array}{l}\text { Mitochondrial } \\
\text { myopathy }\end{array}$ & Colectomy & Appendix \\
\hline
\end{tabular}

disease process, the presence or absence of inflammation, the precise source of the tissue used, and the method of processing. Nevertheless, postnatal human intestine, whether derived from patients with HSCR or without, consistently generates proliferative cells capable of undergoing neuronal and glial differentiation in vitro and in vivo.

To determine the optimal source for obtaining ENSCs, we compared enteric neuronal progenitors isolated from submucosal and myenteric plexuses and found that they possess equivalent neurogenic potential. Previous studies have focused 


\section{Articles | Chenget al.}
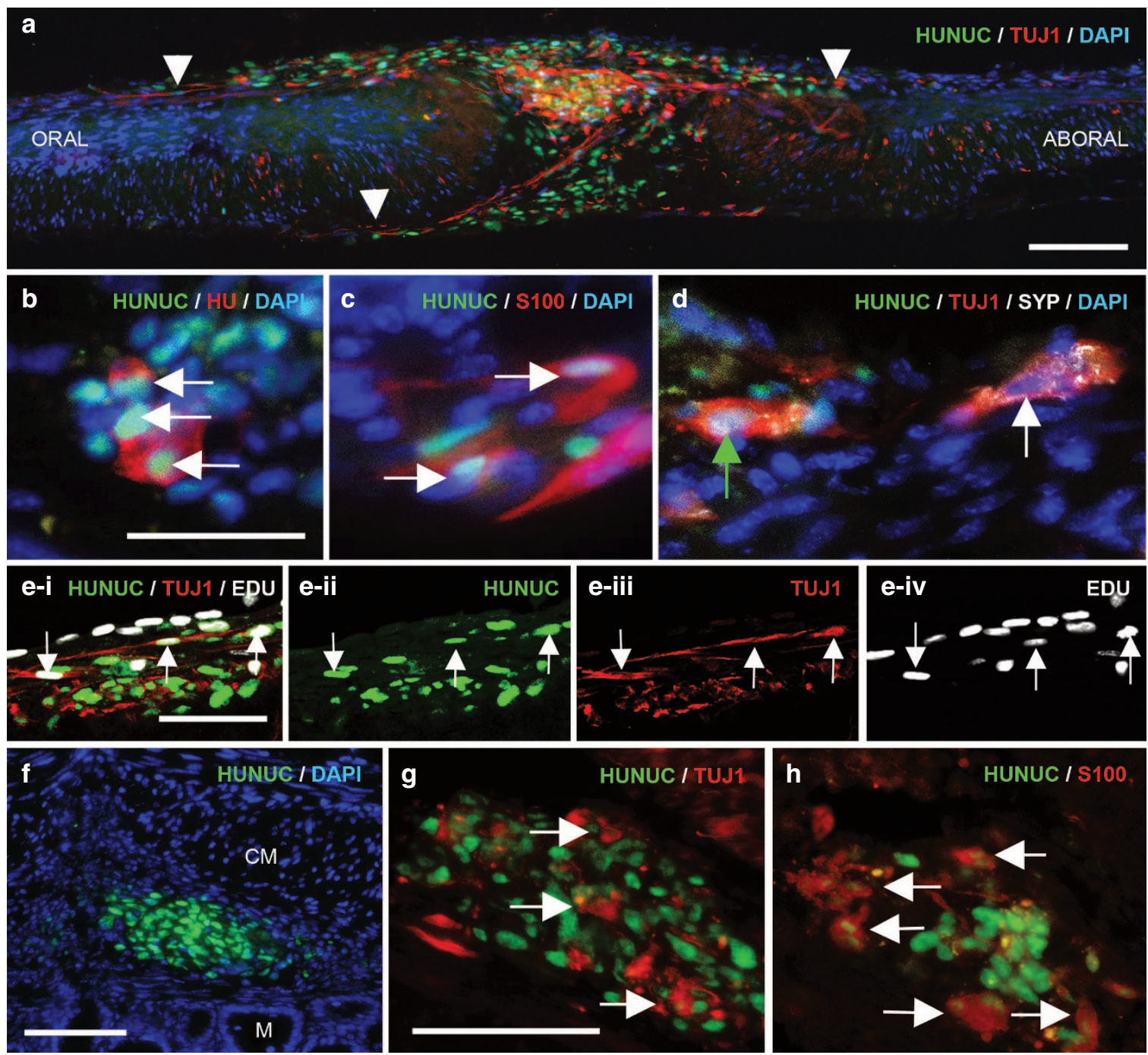

Figure 4. Human ENSCs migrate and differentiate into neurons and glia in aganglionic mouse colon. Human neurospheres were transplanted to explanted muscularis propria isolated from 3-wk-old aganglionic (Ednrb- ${ }^{--}$) or ganglionic (C57BL6) mouse colon and cultured for $7 \mathrm{~d}$. Transplanted human cells coexpressing HUNUC (green) and Tuj 1 (red) migrate extensively along aganglionic colon (a; arrowheads mark farthest extent of migration). Transplanted cells also express markers of neurons (HU, red; $\mathbf{b})$ and glia (S100, red; $\mathbf{c})$. Human Tuj1+ neurons are immunoreactive for synaptophysin (SYP, white, cell nuclei indicated by green arrow; d) in a pattern similar to endogenous neurons (cell nuclei indicated by white arrow; d) and also incorporate EdU (white), indicative of proliferation (e-i to e-iv; arrows mark triple-labeled cells). Human neurospheres were also transplanted to 3-wk-old mouse $\left(E d n r b^{-/-}\right)$aganglionic colon in vivo. After $5 \mathrm{~d}$, transplanted cells are present in the gut wall (f) and express neuronal (TUJ1, red; $\left.\mathbf{g}\right)$ and glial (S100, red; $\mathbf{h}$ ) markers. Cell nuclei are counterstained with DAPI (blue; $\mathbf{a}-\mathbf{d}$, f). Scale bar in $\mathbf{a}=100 \mu \mathrm{m}$. Scale bar in $\mathbf{b}=50 \mu \mathrm{m}$ and applies to $\mathbf{b}-\mathbf{d}$. Scale bar in $\mathbf{e}=50 \mu \mathrm{m}$. Scale bar in $\mathbf{f}=100 \mu \mathrm{m}$. Scale bar in $\mathbf{g}=50 \mu \mathrm{m}$ and applies to $\mathbf{g}$, h.CM, circular muscle; DAPI, 4,6-diamidino-2-2-phenylindole; ENSCs, enteric neural stem/ progenitor cells; $M$, mucosa.

on harvesting ENSCs from the MP $(10,19,20,23)$, but ENSCs can also be harvested from the SP (13). In clinical practice, the $\mathrm{SP}$ is a more attractive source, as cells from this plexus can be harvested endoscopically, thereby avoiding the need for intestinal resection. The ability to isolate cells from both plexuses also enhances the yield of progenitors from harvested tissue. However, others have suggested that the SP may contain a different cell population than the MP, as prior studies in mice have concluded that SP-derived neurospheres contain fewer progenitors and are less neurogenic (17). While we also observed that mouse SP-derived neurospheres contained fewer neural crest-derived progenitors than MP-derived neurospheres, we found no difference in neuronal density or proliferation from
MP- or SP-derived ENSCs in either mice or humans. In contrast to others $(13,17)$, we excluded the mucosal layer when isolating SP neurospheres because it contains a high proportion of highly proliferative nonneural cells, which could reduce the proportion of neural crest-derived cells in the neurospheres. This difference may account for the fact that Becker et al. (17) found that their SP neurospheres contained $0.7 \%$ neural crestderived cells whereas our contained $12.2 \%$.

Compared with induced pluripotent stem cells, postnatalderived ENSC are easily isolated, readily available, and nontumorigenic in long-term studies (24). Autologous ENSCs are an attractive source for cell replacement therapy as they eliminate the need for immunosuppression. However, in patients with 
enteric neuropathies like HSCR, disease-causing mutations may limit the proliferative or neurogenic potential of autologous cells. We previously showed that ENSCs derived from HSCR mice harboring the Ednrb $b^{-/-}$mutation exhibit equivalent migration, proliferation, and neuronal differentiation compared with ENSCs from wild-type mice (14). Similarly, though neuronal survival may be diminished, ENSCs from mice with the $\mathrm{Ret}^{-1-}$ mutation have also been found to have normal migration, proliferation, and differentiation $(15,16,25)$. In this study, we found that the neurogenic potential of ENSCs from patients with and without HSCR was similar, consistent with earlier findings in mice and further supporting the concept of autologous cell transplantation in HSCR. It is possible that some genetic mutations will yield abnormal ENSCs and, in these patients, autologous cell transplantation will require correction of underlying genetic defects prior to auto-transplantation (26). Without knowing the underlying genetic causes of HSCR for the patients in our study, it is impossible to predict if ENSCs from all patients will have the same neurogenic potential. Another limitation of our study was the variability of ENSCs obtained from different patients, which may obscure factors that influence ENSC proliferation and differentiation. We obtained tissue resected for clinical indications and therefore had no control over the age or segment of bowel received. A larger library of human-derived ENSCs is needed in order to strengthen the statistical power of this study and to detect potential differences attributable to patient age, tissue source, and disease process.

Human ENSCs transplanted to embryonic aganglionic intestine may improve gut function $(12,19)$, but there is little information about the ability of these cells to migrate and differentiate in the host environment, particularly in the postnatal gut. We found that postnatal gut-derived ENSCs responded to embryonic cues and behaved like undifferentiated neural crest cells when placed along pathways of normal crest cell migration. The embryonic neural crest and hindgut were highly receptive to the postnatal-derived ENSCs, supporting not only normal patterns of migration, but also pluripotent differentiation into neural crest-derived cell types, including melanocytes. Postnatal aganglionic gut supported neuroglial differentiation of ENSCs, but was less conducive to their migration. Human ENSCs transplanted to postnatal aganglionic mouse colon ex vivo migrated mostly along the surface of the muscularis propria with limited intramural migration in the intermyenteric plane. They also exhibited limited migration from the site of implantation in aganglionic colon in vivo. These findings emphasize the important role of the host microenvironment in guiding the behavior of transplanted cells. The extracellular matrix, which is important in ENS patterning during development (27), may be less permissive to ENSCs postnatally. Comparison of normal and aganglionic gut in human HSCR patients has identified differences in expression of several extracellular matrix proteins (28), suggesting that the extracellular matrix may also play a role in the pathogenesis of HSCR. Postnatal transplantation may be improved by identifying inhibitory or permissive extracellular matrix factors that impede or aid migration of transplanted ENSCs. Further comparison of embryonic and postnatal gut environments may help to elucidate these and other mechanisms that influence ENS development and could be used to facilitate successful engraftment of ENSCs in postnatal recipients.

One limitation of this study is the relatively short duration mice were maintained following cell therapy. Given the fragility and limited survival of the $E d n r b^{-/-}$mouse, especially in the setting of immunosuppression, there was inadequate time to achieve the level of cell engraftment that could feasibly improve gut function. Transplantation of neurospheres, which contain variable numbers and types of cells, also makes it difficult to calculate the rate of engraftment, since the starting number of cells transplanted is difficult to calculate. While we did not observe ganglia formation by transplanted cells, largely because of the short duration following transplantation, the expression of synaptophysin by transplanted ENSCs suggests that functional integration may be occurring. In future studies, another model of aganglionosis, such as experimental aganglionosis induced by benzalkonium chloride (20), may be useful for studying long-term engraftment and functional outcomes. In addition, transplantation of a more precise starting cell population may help better quantify the rates of engraftment and proliferation.

Advances in our understanding of enteric neural progenitors have brought us closer to using cell replacement therapy to treat HSCR and other neurointestinal diseases. Postnatal gutderived ENSCs can be isolated from patients of all ages, from both enteric plexuses, and from patients with and without HSCR. They can successfully engraft into both embryonic and postnatal gut and give rise to new neurons and glia to rebuild the ENS. Further understanding of the role of the microenvironment and its interactions with the ENS will be important to enhance the engraftment and behavior of transplanted ENSCs.

\section{SUPPLEMENTARY MATERIAL}

Supplementary material is linked to the online version of the paper at http:// www.nature.com/pr

\section{ACKNOWLEDGMENTS}

We thank Malkiel Cohen and Rudolf Jaenisch (Massachusetts Institute of Technology, Cambridge, MA) for the kind gift of MITF antibody.

\section{STATEMENT OF FINANCIAL SUPPORT}

L.S.C. is supported by the Society of University Surgeons Ethicon Surgical Research Fellowship Award. R.H. is supported by grants from the Tosteson Fund for Medical Discovery at Massachusetts General Hospital, the REACHirschsprung Foundation, and the American Neurogastroenterology and Motility Society. N.N. is supported by a Bolyai Fellowship from the Hungarian Academy of Sciences. A.M.G. is supported by the National Institutes of Health (R01DK103785).

Disclosures: The authors have no financial or other conflicts of interest to disclose.

\section{REFERENCES}

1. Furness JB. The enteric nervous system and neurogastroenterology. Nat Rev Gastroenterol Hepatol 2012;9:286-94.

2. Goldstein AM, Hofstra RM, Burns AJ. Building a brain in the gut: development of the enteric nervous system. Clin Genet 2013;83:307-16.

3. Ieiri S, Nakatsuji T, Akiyoshi J, et al. Long-term outcomes and the quality of life of Hirschsprung disease in adolescents who have reached 18 years or 


\section{Articles | Chenget al.}

older-a 47-year single-institute experience. J Pediatr Surg 2010;45:2398402.

4. Neuvonen MI, Kyrklund K, Rintala RJ, Pakarinen MP. Bowel function and quality of life after transanal endorectal pull-through for Hirschsprung disease: Controlled outcomes up to adulthood. Ann Surg 2016. doi:10.1097/ SLA.0000000000001695.

5. Burns AJ, Thapar N. Neural stem cell therapies for enteric nervous system disorders. Nat Rev Gastroenterol Hepatol 2014;11:317-28.

6. Hotta R, Natarajan D, Thapar N. Potential of cell therapy to treat pediatric motility disorders. Semin Pediatr Surg 2009;18:263-73.

7. Wilkinson DJ, Edgar DH, Kenny SE. Future therapies for Hirschsprung's disease. Semin Pediatr Surg 2012;21:364-70.

8. Rauch U, Hänsgen A, Hagl C, Holland-Cunz S, Schäfer KH. Isolation and cultivation of neuronal precursor cells from the developing human enteric nervous system as a tool for cell therapy in dysganglionosis. Int J Colorectal Dis 2006;21:554-9.

9. Almond S, Lindley RM, Kenny SE, Connell MG, Edgar DH. Characterisation and transplantation of enteric nervous system progenitor cells. Gut 2007;56:489-96.

10. Metzger M, Bareiss PM, Danker T, et al. Expansion and differentiation of neural progenitors derived from the human adult enteric nervous system. Gastroenterology 2009;137:2063-2073.e4.

11. Lindley RM, Hawcutt DB, Connell MG, Edgar DH, Kenny SE. Properties of secondary and tertiary human enteric nervous system neurospheres. J Pediatr Surg 2009;44:1249-55; discussion 1255-6.

12. Wilkinson DJ, Bethell GS, Shukla R, Kenny SE, Edgar DH. Isolation of enteric nervous system progenitor cells from the aganglionic gut of patients with Hirschsprung's Disease. PLoS One 2015;10:e0125724.

13. Metzger M, Caldwell C, Barlow AJ, Burns AJ, Thapar N. Enteric nervous system stem cells derived from human gut mucosa for the treatment of aganglionic gut disorders. Gastroenterology 2009;136:2214-25.e1-3.

14. Hotta R, Cheng LS, Graham HK, et al. Isogenic enteric neural progenitor cells can replace missing neurons and glia in mice with Hirschsprung disease. Neurogastroenterol Motil 2016;28:498-512.

15. Iwashita T, Kruger GM, Pardal R, Kiel MJ, Morrison SJ. Hirschsprung disease is linked to defects in neural crest stem cell function. Science 2003;301:972-6.

16. Bondurand N, Natarajan D, Thapar N, Atkins C, Pachnis V. Neuron and glia generating progenitors of the mammalian enteric nervous system iso- lated from foetal and postnatal gut cultures. Development 2003;130:6387400.

17. Becker L, Kulkarni S, Tiwari G, Micci MA, Pasricha PJ. Divergent fate and origin of neurosphere-like bodies from different layers of the gut. Am J Physiol Gastrointest Liver Physiol 2012;302:G958-65.

18. Hagl CI, Heumüller-Klug S, Wink E, Wessel L, Schäfer KH. The human gastrointestinal tract, a potential autologous neural stem cell source. PLoS One 2013;8:e72948.

19. Lindley RM, Hawcutt DB, Connell MG, et al. Human and mouse enteric nervous system neurosphere transplants regulate the function of aganglionic embryonic distal colon. Gastroenterology 2008;135:205-216.e6.

20. Hetz S, Acikgoez A, Voss U, et al. In vivo transplantation of neurospherelike bodies derived from the human postnatal and adult enteric nervous system: a pilot study. PLoS One 2014;9:e93605.

21. Hosoda K, Hammer RE, Richardson JA, et al. Targeted and natural (piebald-lethal) mutations of endothelin-B receptor gene produce megacolon associated with spotted coat color in mice. Cell 1994;79:1267-76.

22. Hotta R, Cheng LS, Graham HK, et al. Delivery of enteric neural progenitors with 5-HT4 agonist-loaded nanoparticles and thermosensitive hydrogel enhances cell proliferation and differentiation following transplantation in vivo. Biomaterials 2016;88:1-11.

23. Grundmann D, Klotz M, Rabe H, Glanemann M, Schäfer KH. Isolation of high-purity myenteric plexus from adult human and mouse gastrointestinal tract. Sci Rep 2015;5:9226.

24. Cooper JE, McCann CJ, Natarajan D, et al. In vivo transplantation of enteric neural crest cells into mouse gut; engraftment, functional integration and long-term safety. PLoS One 2016;11:e0147989.

25. Uesaka T, Nagashimada M, Yonemura S, Enomoto H. Diminished Ret expression compromises neuronal survival in the colon and causes intestinal aganglionosis in mice. J Clin Invest 2008;118:1890-8.

26. Fattahi F, Steinbeck JA, Kriks S, et al. Deriving human ENS lineages for cell therapy and drug discovery in Hirschsprung disease. Nature 2016;531:1059.

27. Nagy N, Barad C, Graham HK, et al. Sonic hedgehog controls enteric nervous system development by patterning the extracellular matrix. Development 2016;143:264-75.

28. Zhang SC, Chen F, Jiang KL, Yuan ZW, Wang WL. Comparative proteomic profiles of the normal and aganglionic hindgut in human Hirschsprung disease. Pediatr Res 2014;75:754-61. 